FACULDADE DE CIÊNCIAS ECONô MICAS dA UFRGS
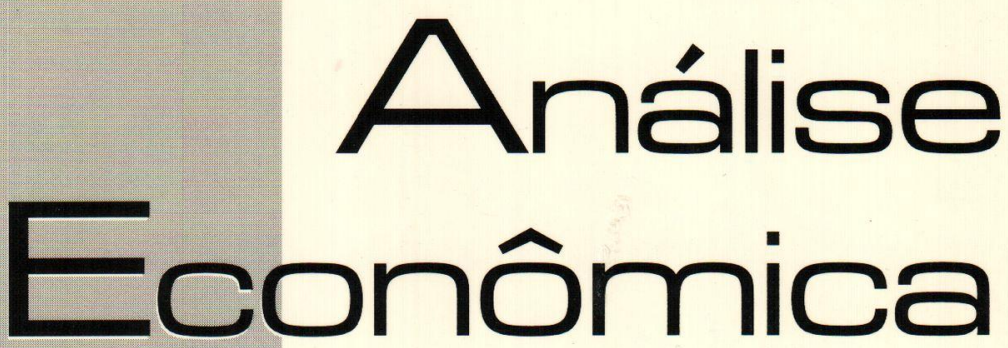

ESCOLHA DE PORTFÓLIO, INVESTIMENTO E NĀO. NEUTRALIDADE DA MOEDA

JOSÉ LUIS OREIRO

REFORMAS NA AROUITETURA FINANCEIRA INTERNACIONAL: NOVIDADES NO FRONT? ANDRÉ MOREIRA CUNHA

MERCOSUR'S CHANGE IN TRADE PATTERNS ANDRÉ FILIPE ZAGO DE AZEVEDO

O IMPACTO DA COMPOSICÃO SETORIAL, DOS FLUXOS INTRA-SETORIAIS E DA ABERTURA COMERCIAL NA PARTICIPAÇÁO DE MERCADO DAS EXPORTAÇÓES BRASILEIRAS

CLÉSIO LOURENÇO XAVIER E EMERSON FERNANDES MARÇAL

O EFEITO BALASSA-SAMUELSON E A PARIDADE DO PODER DE COMPRA NA ECONOMIA BRASILEIRA CLÁUDIO ROBERTO FÓFFANO VASCONCELOS

CICLOS Y FLUCTUACIONES FINANCIERAS: LA IRREGULAR DINÁMICA ECONÓMICA

SARY LEVY-CARCIENTE

O PENSAMENTO DE KARL POPPER: AS DIFERENTES INTERPRETACÓES DOS METODÓLOCOS DA CIENCIA ECONÓMICA

SOLANCE REGINA MARIN E RAMÓN GARCÍA FERNÁNDEZ

A EXPANSÃO DO ESCOPO TEMÁTICO DAS NEGOCIAÇÓES COLETIVAS DE TRABALHO CARLOS HENRIQUE HORN

EFEITOS DO CAPITAL SOCIAL E DO CAPITAL POLITICO NO DESENVOLVIMENTO ECONOMMICO SIMULAÇOES PARA PAISES E ESTADOS BRASILEIROS

RONALDO A. ARRAES, RICARDO CANDÉA S. BARRETO E VLADIMIR KÜHL TELES

O PROBLEMA DE RISCO MORAL NO MERCADO BRASILEIRO DE ASSISTÊNCIA MÉDICA SUPLEMENTAR LUCIANA PINTO DE ANDRADEE SABINO DA SILVA PÓRTO JÚNIOR

ANO

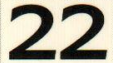

No 41

Março, 2004 
Universidade Federal do Rio Grande do Sul

Reitora: Profo. Wrana Maria Panizzi

Faculdade de Ciencias ECONOMICAS

Diretora: Prof Pedro César Dutra Fonseca

Centro de Estudos e Pesquisas Economicas

Diretor: Prof. Gentil Corazza

Departamento de Ciencias Económicas

Chefe: Prof. Ricardo Dathein

Curso de Pós-Graduação em Economia

Coordenador: Prof. Eduardo Pontual Ribeiro

Programa de Pó-Graduação em Desenvolvimento Rural

Coordenador: Prof. Jalcione Almeida

CONSElHo EDITORIAL:

Carlos G. A. Mielitz Netto (UFRGS), Eduardo A. Maldonado Filho (UFRGS), Eduardo P. Ribeiro (UFRGS), Eleutério F. S. Prado (USP), Eugênio Lagemann (UFRGS), Fernando Cardim de Carvalho (UFRJ), Fernando Ferrari Filho (UFRGS), Fernando de Holanda Barbosa (FGV/RJ), Flávio Vasconcellos Comim (UFRGS), Gentil Corazza (UFRGS), Giácomo Balbinotto Netto (UFRGS), Gustavo Franco (PUC/RJ), Jan A. Kregel (UNCTAD), João Rógério Sanson (UFSC), Joaquim Pinto de Andrade (UnB), Jorge Paulo Araújo (UFRGS), Juan H. Moldau (USP), Marcelo S. Portugal (UFRGS), Maria Alice Lahorgue (UFRGS), Paul Davidson (University of Tennessee), Paulo D. Waquil (UFRGS), Pedro C. D. Fonseca (UFRGS), Philip Arestis (Levy Economics Institut of Bard College), Roberto C. de Moraes (UFRGS), Ronald Otto Hillbrecht (UFRGS), Sabino da Silva Porto Jr. (UFRGS), Stefano Florissi (UFRGS) e Werner Baer (University of Illinois at Urbana-Champaign).

COMISSĀO EDITORIAL:

Eduardo Augusto Maldonado Filho, Fernando Ferrari Filho, Gentil Corazza, Marcelo Savino Portugal, Paulo Dabdab Waquil e Roberto Camps Moraes.

EDITOR: Prof. Fernando Ferrari Filho

Editor Adjunto: Prof. Gentil Corazza

SeCretária: Paulo Roberto Eckert

REVISÃO DE TEXTOS: Vanete Ricacheski

EdITORAÇÃo Eletrónca: Vanessa Hoffmann de Quadros

Fundador: Prof. Antônio Carlos Santos Rosa

Os materiais publicados na revista Análise Econômica são da exclusiva responsabilidade dos autores. É permitida a reprodução total ou parcial dos trabalhos, desde que seja citada a fonte. Aceita-se permuta com revistas congêneres. Aceitam-se, também, livros para divulgação, elaboração de resenhas e recensões. Toda correspondência, material para publicaçāo (vide normas na terceira capa), assinaturas e permutas devem ser dirigidos ao seguinte destinatário:

PROF. FERNANDO FERRARI FILHO Revista Análise Econômica - Av. João Pessoa, 52 CEP 90040-000 PORTO ALEGRE - RS, BRASIL Telefones: (051) 316-3513 - Fax: (051) 316-3990

Análise Econômica E-mail: rae@ufrgs.br

Ano 22, $n^{\circ} 41$, março, 2004 . Porto Alegre

Faculdade de Ciências Econômicas, UFRGS, 2004

Periodicidade semestral, março e setembro.

Tiragem: 500 exemplares

1. Teoria Econômica - Desenvolvimento Regional -

Economia Agrícola - Pesquisa Teórica e Aplicada -

Periódicos. I. Brasil

Faculdade de Ciências Econômicas,

Universidade Federal do Rio Grande do Sul. 


\title{
O efeito Balassa-Samuelson e a Paridade do Poder de Com* pra na economia brasileira
}

\author{
Cláudio Roberto Fóffano Vasconcelos*
}

\begin{abstract}
Resumo: Dada a importância da hipótese da paridade de poder de compra (PPC) nos modelos de determinação da taxa de câmbio, no presente artigo objetivou-se verificar empiricamente a crítica a esta teoria em termos do crescimento econômico afetando os preços relativos, ou seja, testar o efeito Balassa-Samuelson para a economia brasileira, no período entre 1980 a 2001 . Utilizando uma abordagem de séries temporais, através do teste de fronteira para a análise de relàcionamento de longo prazo desenvolvido por Pesaran, Shin e Smith (1999), verificou-se se as variáveis razão dos níveis de preços e razão do nível de renda real per capita cointegram. Os resultados do trabalho evidenciaram que não existe relação de longo prazo entre as variáveis, isto é, não há evidência do efeito Balassa-Samuelson para a economia brasileira no período considerado.

Palavras-Chave: Efeito Balassa-Samuelson; Preços relativos; Renda real; Paridade de poder de compra.
\end{abstract}

Abstract: Given the importance of the purchasing power parity (PPP) hypothesis of the models of exchange rate determination, this paper aims to verify empirically the economic growth affecting the relative prices. In others words, we tested the Balassa-Samuelson effect for the brazilian economy, between the years of 1980 to 2001. It was applied the temporal series methodology, using on bounds testing approache to the analysis of long run relationships developed by Pesaran, Shin and Smith (1999), to verify the existence of a cointegration vector between the price ratio and per capita real income ratio. The results do not show supportive evidence for the Balassa-Samuelson effect for the brazilian economy in this period.

Keywords: Balassa Samuelson Effect; Relate prices; Real income; Purchasing power parity.

JEL: F10; F11

\footnotetext{
- Prof Adjunto da Fundação Universidade Federal do Rio Grande. Avenida Itália km 8, Campus Carreiros, Rio Grande -RS. CEP 96201-900. e-mail: dceaccrv@furg.br. O autor agradece à Fapergs o apoio ARD.
} 


\section{Introdução}

Historicamente, a comprovação empírica da paridade de poder de compra (PPC) tem despertado um grande interesse no meio científico, visto que alguns modelos macroeconômicos de determinação da taxa de câmbio têm como hipótese a validade da PPC. Isto é, modelos de determinação da taxa de câmbio como, por exemplo, a abordagem monetarista, a teoria da PPC figura como um pressuposto fundamental, visto que, nesta abordagem, se trabalha com a hipótese de preços flexíveis. A verificação empírica de validade da hipótese da teoria da paridade do poder de compra implica, conseqüentemente, a análise da existência de uma taxa de câmbio de equilibrio de longo prazo'.

Neste contexto, Balassa (1964) e Samuelson (1964), preocupados em explicar os persistentes desvios da PPC, estabeleceram um modelo relacionando os movimentos da taxa de câmbio real de longo prazo e os preços relativos dos bens não comercializáveis (nontradables).

Criticando a premissa da PPC como uma mensuração da taxa de câmbio de equilibrio de longo prazo, Balassa (1964) argumentou que, se as diferenças de produtividade internacionais são maiores ou crescem a uma taxa maior na produção de bens comercializáveis (tradables) do que na produção de não-comercializáveis (nontradables), o preço relativo destes últimos tenderá a crescer devido ao fato de ambos competirem por fatores domésticos de produção. Como resultado, a moeda do país com nível de produtividade mais alta aparecerá sobrevalorizada em termos da PPC. Isto é, considerando que os preços dos tradables tendem a se igualarem no mercado internacional, um aumento no preço relativo de nontradables causa uma sobrevalorização em termos da PPC. Este seria, então, o chamado efeito Balassa-Samuelson.

Como salientam De Gregório e Wolf (1994), o argumento de diferencial de produtividade setorial entre países tem sido utilizado ao longo do tempo como um dos principais determinantes do movimento da taxa de câmbio real.

' Com relação ao teste empírico de validade da PPC para a economia brasileira, ver VASCON. CELOS et alii (1999). 
Portanto, como a taxa de câmbio constitui-se num dos preços fundamentais da economia, objetiva-se com este trabalho verificar empiricamente uma das críticas à PPC, através do argumento de crescimento econômico afetando os preços relativos internos. Para tanto, é feito um teste de cointegração sobre a validade do efeito BalassaSamuelson para a economia brasileira, considerando o período entre 1980 e 2001 . Como se trabalha com níveis de preços e renda per capita, a ocorrência de quebras estruturais nestas séries, decorrentes dos vários planos econômicos implementados, poderia mascarar a ordem de integração das variáveis. Desta forma, com o objetivo de contornar este problema, empregou-se a abordagem do teste de fronteira de Pesaran, Shin e Smith (1999). Esta metodologia permite testar a existência de um relacionamento de longo prazo entre a variável dependente e um conjunto de regressores quando a ordem das variáveis envolvidas é incerta.

Dadas estas considerações, o restante do trabalho está dividido da seguinte forma: a seção 2 traz alguns trabalhos empíricos sobre a validade do efeito Balassa-Samuelson. Na seção 3, descreve-se o modelo utilizado e na 4, o modelo econométrico. Nas seções 5 e 6 , estão os resultados obtidos e as considerações finais, respectivamente.

\section{Evidências empíricas do efeito Balassa-Samuelson}

Inicialmente, o modelo de Balassa-Samuelson de diferença de produtividade setorial entre países pressupõe um mercado de trabalho competitivo, com perfeita mobilidade em cada país; um mercado competitivo para bens tradables; e validade da PPC somente para bens tradables. As implicações destas pressuposições são que, para o mercado interno, a mobilidade do trabalho leva à equalização dos salários entre os setores de bens tradables e nontradables. Sendo válida a PPC para tradables, o crescimento nos preços externos implica um crescimento idêntico nos preços domésticos dos tradables, logo o diferencial de produtividade entre trabables e nontradables determinaria os seus preços relativos.

De forma alternativa ao modelo de diferença de produtividade, uma justificativa para a relação entre nível de preços e renda pode ser obtida na teoria das dotações fatoriais. Considere um modelo com dois bens, dois fatores, dois países, onde o setor produtor de 
nontradables seja relativamente intensivo em trabalho e o setor de tradables intensivo em capital. Assim, a abundância relativa de capital de um país rico irá lhe propiciar uma vantagem comparativa na produção de tradables. Neste sentido, o nível de preços dos nontradables relativamente aos tradables, e conseqüentemente o nível de preços internos, irá ser maior nos países com alta renda per capita (BERGSTRAND,1991, p. 326).

Analisando o nível de preços para tradables e nontradables entre países ao longo do tempo, e relacionando o efeito de variações no nível de preços nas mudanças do nível de renda, Kravis e Lipsey (1988) concluíram que, entre países, o nível de preços nacional aumenta sistematicamente com o nível de renda per capita dos países e que a razão de preços entre tradables e nontradables decresce. Desta forma, os autores estariam confirmando o que prediz o modelo de Balassa-Samuelson.

Nesta linha, Faria e León-Ledesma (2000) verificam a existência de uma relação de longo prazo entre a taxa de câmbio real (razão dos níveis de preços internos e externos) e o diferencial de produtividade entre países (razão do nível de renda real per capita entre países). Considerando quatro países desenvolvidos (Alemanha, Estados Unidos, Inglaterra e Japão), para informações entre 1960 e 1996, os autores concluíram que o efeito Balassa-Samuelson no longo prazo não se confirmou para o conjunto de países analisados, ou seja, as séries analisadas não apresentaram evidencias de uma relação de longo prazo (não cointegram) entre a taxa de câmbio e a diferença de produtividade.

Ao contrário das abordagens anteriores, que consideram o lado da oferta (modelo de diferencial de produtividade ou hipótese de dotações relativas de fatores), Bergstrand (1991) estuda a relação entre o nível de preços domésticos e taxa de câmbio real enfocando o lado da demanda. Segundo o autor, assumindo gostos nãohomotéticos, países com altos níveis de renda per capita irão exibir, em equilíbrio, forte demanda por bens nontradables relativamente a tradables, aumentando seu nível de preços. Neste sentido, o autor conclui que empiricamente há uma significativa correlação positiva entre o nível de preços interno e o nível de produto (renda real per capita). Desta forma, em uma análise pelo lado da demanda, confirma-se empiricamente as implicações do modelo de diferenças de produtividade de Balassa e Samuelson. 
De Gregoria e Wolf (1994), considerando uma amostra de 14 países da OECD, para o período de 1970 a 1985, examinam o efeito dos termos de troca e diferenças de produtividade entre setores e o comportamento da taxa de câmbio real. Os autores concluíram que tanto as flutuações nos termos de troca quanto o diferencial de crescimento da produtividade entre setores são altamente significantes na determinação dos movimentos da taxa de câmbio real. Além disto, os termos de troca afetam a taxa de câmbio real principalmente através do efeito renda.

Já Macdonald e Ricci (2001) investigam o papel do setor de distribuição na explicação dos desvios da taxa de câmbio real em relação à PPC. A idéia central desta abordagem é que, quando se considera o setor de distribuição como pertencente ao setor produtor de bens nontradables, um aumento na eficiência relativa do setor de distribuição, com respeito a um país estrangeiro, acarretará em preços menores para os serviços de distribuição e, conseqüentemente, em um menor índice de preços internos, comparativamente ao país estrangeiro. Isto induziria a uma depreciação da taxa de câmbio real.

Desta forma, procurando testar esta hipótese, os autores utilizam a metodologia de dados em painel dinâmico (dynamic panel data) para estabelecer a relação de longo prazo entre a taxa de câmbio real e a eficiência do setor de distribuição para dez países industrializados ${ }^{2}$, o período considerado foi de 1970 a 1992. A principal conclusão dos autores foi a de que um aumento na produtividade no setor de distribuição com respeito a países estrangeiros leva a uma apreciação da taxa de câmbio real. Este resultado contrastaria com o esperado, visto que se esperaria uma depreciação e não uma apreciação cambial. Entretanto, este resultado evidenciaria que o aumento da eficiência relativa do setor de distribuição decorre dos serviços do setor de distribuição de tradables. Assim, o resultado encontrado é similar aos prognósticos do modelo de diferenças de produtividade para um aumento relativo na produtividade doméstica no setor de tradables.

Em outra linha de investigação, Strauss (1998) testa ${ }^{3}$ as pressuposições do modelo de diferenças na produtividade Balassa-

${ }^{2}$ Bélgica, Dinamarca, Finlândia, França, Itália, Japão, Noruega, Suécia, Alemanha Ocidental e Estados Unidos. 
Samuelson. As hipóteses são as de mobilidade e competitividade no mercado de trabalho (acarretando em equalização de salários reais entre setores) e a validade da PPC para o setor de bens tradables. Segundo o autor, os resultados evidenciaram tanto a rejeição das hipóteses de equalização dos salários reais entre setores de tradables e nontradables quanto o relacionamento entre produtividade e taxa de crescimento do salário real. A conclusão é que o modelo de Balassa-Samuelson de diferenças na produtividade, que explica os movimentos nos preços relativos, ignoraria uma importante variável explicativa, a diferença de salários.

Com relação à economia brasileira, na literatura consultada não foi encontrada verificação empírica direta do efeito BalassaSamuelson. Figuram apenas trabalhos empíricos referentes à validade da $\mathrm{PPC}^{4}$, apesar de a literatura não ser muito extensa.

Neste contexto, Pereira e Duarte (1991), utilizando informações mensais para o período de 1981 até 1988 e metodologia de cointegração em um modelo multivariado de correção de erros (procedimento de Johansen), testam a validade da PPC e paridade da taxa de juros concomitantemente. $O$ trabalho conclui que, para $O$ teste conjunto de PPC e paridade da taxa de juros, há evidências de existir vetores cointegrados. Logo, há evidência de validade da PPC.

Menezes e Resende (1996) procederam a um teste de validade da PPC para o Brasil, baseado em um teste de cointegração para o período pré-industrial, abrangendo o período de 1870 a 1906 . O trabalho conclui que os testes não confirmaram a validade da PPC. Entretanto, segundo os autores, o resultado encontrado não pode ser considerado como uma resposta final, porque depende do índice de preço usado para a economia brasileira, visto que pode existir algum viés com respeito a bens não comercializáveis no mercado internacional.

Zini e Cati (1993), trabalhando série de dados abrangendo o período de 1855 a 1990 , encontram resultados que evidenciam a rejeição da hipótese da validade da PPC. Isto é, pelos testes realizados, não se pode rejeitar a hipótese de caminhada aleatória como modelo para a taxa de câmbio real no longo prazo.

${ }^{3}$ Em uma análise de curto e médio prazos para as economias da França, Alemanha, Japão, Reino Unido e Estados Unidos, abrangendo o período entre 1960-90.

${ }^{4}$ Que seria uma forma indireta de se verificar o efeito Balassa-Samuelson. 
De forma semelhante, Fava e Alves (1996), a partir da metodologia de cointegração fracional para a mesma série histórica utilizada por Zini e Cati (1993), chegam à conclusão de que a análise de cointegração da regressão de cointegração residual indica a presença de raiz unitária. Portanto, se rejeita a validade da PPC como explicação para ajustamentos da taxa de câmbio no Brasil para o período analisado.

Contrapondo estes dois últimos trabalhos, Rossi (1996) aplica o teste de cointegração, baseado no modelo de Johansen para a economia brasileira. O autor conclui que, com a utilização do índice de preços por atacado (ou seja, considerando apenas os bens tradables), os resultados indicam que existem dois vetores cointegrados, e quando se utiliza o índice geral de preços (considerando tanto tradables quanto nontradables), há apenas um vetor cointegrado. Ou seja, em ambos os casos, foi confirmada a validade da PPC para a economia brasileira, no período em análise.

Assim como Rossi (1996), Vasconcelos et alii (1999) confirmaram a validade da PPC, considerando o período entre 1980: 1 a 1994:6, utilizando o índice de preços por atacado. Estes resultados, para a economia brasileira, são coerentes com estudos empíricos de outras economias. Pois, em economias caracterizadas por altos processos inflacionários, a validade da PPC se verifica de forma mais fácil.

\section{0 efeito Balassa-Samuelson 5}

Sejam dois países (doméstico e estrangeiro) que usam o fator de produção trabalho (L) para produzir dois tipos de bens, um tradable $(\mathrm{T})$ e outro nontradable $(\mathrm{N})$. A função de produção para estes dois bens apresenta retornos constantes de escala.

As pressuposições com relação ao mercado de trabalho são as de que ele é competitivo e perfeitamente móvel entre setores, mas não entre países. Portanto, há equalização do salário real entre os setores de um mesmo país. Então:

$$
\begin{aligned}
& P_{T} f^{\prime}\left(L_{T}\right)=w=P_{N} g^{\prime}\left(L_{N}\right) \\
& P_{T}^{*} F^{\prime}\left(L_{T}^{*}\right)=w^{*}=P_{N}^{*} G^{\prime}\left(L_{N}^{*}\right)
\end{aligned}
$$

${ }^{5}$ Esta seçāo esta baseada em ANDRÉS et alli (1996) e FARIA e LEÓN-LEDESMA (2000). 
onde $P_{T}$ e $P_{N}$ são preços dos bens tradables e nontradables; wé o salário real; $P_{T}^{*}, P_{N}^{*}$ e $w^{*}$ estes mesmos valores para o país estrangeiro; $f^{\prime}$ e $g^{\prime}, F^{\prime}$ e $G^{\prime}$ representam o produto marginal do trabalho para os setores de tradable e nontradable do país doméstico e estrangeiro, respectivamente.

Como o modelo de diferenças de produtividade de BalassaSamuelson pressupõe validade da PPC para o setor de bens tradable, então:

$$
P_{T}=e P_{T}^{*}
$$

onde e denota a taxa de câmbio nominal.

Considerem-se, também, os níveis de preços interno $(P)$ e externo $\left(P^{*}\right)$ como uma média geométrica ponderada dos preços de ambos os setores, isto é:

$$
\begin{aligned}
& P=P_{T}^{1-i} P_{N}^{i} \\
& P^{*}=P_{T}^{* 1-j} P_{N}^{* j}
\end{aligned}
$$

onde $i$ e $j$ indicam os pesos do setor de nontradables na produção total doméstica e estrangeira, respectivamente.

Normalizando os preços para o setor de bens tradables, $P_{T}=P_{T}^{*}=1$, as equações para os níveis de preços doméstico e estrangeiro ficam:

$$
\begin{aligned}
& P=P_{N}^{i} \\
& P^{*}=P_{N}^{* j}
\end{aligned}
$$

De forma similar, empregando a normalização dos preços do setor de tradables, as equações (1) e (2) ficam:

$$
\begin{aligned}
& P_{N}=f^{\prime}\left(L_{T}\right) / g^{\prime}\left(L_{N}\right) \\
& P_{N}^{*}=F^{\prime}\left(L_{T}^{*}\right) / G^{\prime}\left(L_{N}^{*}\right)
\end{aligned}
$$

Considere a taxa de câmbio real definida como $\theta=\frac{P}{e P^{*}}$. Assim, substituindo as equações (6) e (7) nas equações (4) e (5) e o resultado na taxa de câmbio real, tem-se: 


$$
\theta=\frac{P}{P^{*}}=\frac{\left[f^{\prime}\left(L_{T}\right) / g^{\prime}\left(L_{N}\right)\right]^{i}}{\left[F^{\prime}\left(L_{T}^{*}\right) / G^{\prime}\left(L_{N}^{*}\right)\right]^{j}}
$$

Desta forma, a equação (8) retrataria o efeito Balassa-Samuelson. Observe que, de acordo com a equação (8), se o numerador está crescendo à taxa superior à do denominador, implica um aumento da taxa de câmbio real. Em outras palavras, um crescimento mais rápido da produtividade do setor de tradables no país doméstico comparativamente ao país estrangeiro acarretaria uma apreciação da taxa de câmbio real. Portanto, existiria um link positivo entre o crescimento da produtividade do setor de tradables e a taxa de câmbio real.

\section{Modelo econométrico}

Considerando a pressuposição de retornos constantes à escala, a produtividade marginal do trabalho é proporcional ao produto médio do trabalho. Portanto, o lado direito da equação (8) pode ser reescrito em termos da produtividade média do trabalho.

Segundo Faria e León Ledesma (2000), tratando o crescimento da produtividade média do trabalho como crescimento da renda real per capita, podem-se estabelecer duas especificações testáveis da forma reduzida do modelo. Ou seja:

$$
\begin{aligned}
& P_{(A B) t}=\alpha_{1}+\beta_{1} Y_{(A B) t}+u_{t} \\
& P_{(A B) t}=\alpha_{2}+\beta_{2} \Delta Y_{(A B) t}+v_{t}
\end{aligned}
$$

Onde, $P_{(A B)}$ é o logaritmo da razão entre os índices de preços do país doméstico (A) com relação ao país estrangeiro (B); o $Y_{(A B)}$ é o logaritmo da razão entre os níveis reais de renda per capita do país $A$ e B; e $\Delta Y_{(A B)}$ é a variação do logaritmo da razão dos níveis reais de renda per capita do país $\mathrm{A}$ e $\mathrm{B}$.

As equaçōes (9) e (10) expressariam, então, o relacionamento de longo prazo entre a razão dos preços internos e externos e sua razão do produto per capita. Desta forma, se existir um vetor de cointegração de longo prazo para os modelos propostos, pode-se inferir que o efeito Balassa Samuelson se confirma, para um dado período de tempo, com relação a dadas duas economias.

De acordo com a abordagem tradicional das técnicas de cointegração, o primeiro passo para a verificação da possível exis- 
tência de um (ou mais) vetor de cointegração é a verificação da ordem de integração das variáveis. Entretanto, como se está trabalhando com as variáveis como níveis de preços, renda e, principalmente, na equação (10) uma variável em nível e outra na primeira diferença, as diferentes ordens de integraçāo das variáveis podem comprometer os resultados.

Neste sentido,. Pesaran et alii (1999) propõem uma abordagem alternativa para testar a existência de uma relação de longo prazo entre duas ou mais variáveis sem, necessariamente, ter que se estabelecer a ordem de integração destas variáveis.

A idéia central do teste de fronteira desenvolvido por Pesaran et alii (1999) é a de que, através da estimação de um modelo de representação dinâmica de correção dos erros ${ }^{6}$, é possível testar se os níveis defasados das variáveis são significativos. Através da estatística F, usada para testar a significância das variáveis defasadas em uma regressão na primeira diferença, é possível construir dois conjuntos (extremos) assintóticos de valores críticos. Um conjunto (extremo superior) assume que todos os regressores são integrados de ordem um, I(1), e outro (extremo inferior) assume que não são integrados, I(0). Desta forma, estes dois conjuntos de valores críticos cobririam todas as possibilidades de classificação dos regressores em I(0), I(1) ou mutuamente integrados.

Formalmente, Pesaran et alii (1999), partindo de um vetor autoregressivo de ordem $\mathrm{p}(\operatorname{VAR}(\mathrm{p}))$, chegam ao seguinte modelo de correção de erros (ECM):

$$
\Delta y_{t}=a_{0}+a_{1} t+b_{1} y_{t-1}+b_{2} x_{t-1}+\sum_{i=1}^{p-1} \gamma \Delta y_{t-i}+\sum_{i=0}^{p-1} \varphi \Delta x_{t-i}+\mu_{t}
$$

Confirma-se o equilíbrio de longo prazo entre as variáveis, se b1 e b2 conjuntamente são diferentes de zero. Quando o modelo possui um tendência determinística, o teste $\mathrm{F}$ inclui também a hipótese nula de que $a_{1}=0$.

Segundo os autores, o teste é para a falta de alguma relação de longo prazo entre as variáveis. A hipótese nula do teste contempla, portanto, a possibilidade de que não exista um relação de longo

\footnotetext{
${ }^{6} \mathrm{Na}$ forma de um teste $\mathrm{F}$ para uma regressão do tipo Dickey-Fuller aumentada, usada para testar o nível de significância das variáveis sob a condição de uma regressão de erros irrestrito.
} 
prazo entre a variável dependente e as variáveis independentes, a despeito da ordem de integração das variáveis. Assim, se o valor calculado da estatística $F$ for menor ou maior do que os valores críticos inferiores e superiores (fronteira) ${ }^{7}$, respectivamente, uma conclusiva inferência pode ser tirada sem a necessidade de se conhecer a ordem de integração das variáveis envolvidas. Isto é, se o valor do teste de hipótese conjunta, teste $\mathrm{F}$, calculado for menor que o valor crítico para $\mathrm{I}(0)$, pode-se inferir que não existe uma relação de longo prazo entre as variáveis, independentemente dos regressores serem $\mathrm{I}(0)$ ou $\mathrm{I}(1)$. Caso contrário, se o $\mathrm{F}$ calculado for maior que o valor crítico para $\mathrm{I}(1)$, a hipótese de que não existe relação de longo prazo é rejeitada. Por fim, se o valor calculado de $\mathrm{F}$ for maior que o valor crítico para $\mathrm{I}(0)$ e menor que o valor crítico I(1), a inferência pode ser inconclusiva. Neste caso, será necessário conhecer a ordem de integração das variáveis envolvidas antes de se fazer alguma afirmação.

\section{Resultados}

Para a aplicação empírica do modelo foram consideradas informações trimestrais de nível de preços e renda real per capita para as economias do Brasil e Estados Unidos da América, referente ao período de 1980:1 a 2001:1. As informaçōes empregadas foram o deflator implícito de preços para o nível de preços $^{8}$ (BEA, 2002; e IBGE, 1989 e 2001), PIB e PNB, e população para o nível de renda real per capita ${ }^{9}$ para as economias brasileira e americana, respectivamente (BEA, U. S. CENSUS BUREAU, e IPEA, 2002; e IBGE 1989 e 2001). Estes valores têm como base o ano de 1996:4.

\footnotetext{
${ }^{7}$ As Tabelas C1.i a C1.v em PESARAN et alii (1999, p. Tl a T3) provem dois conjuntos de valores críticos assintóticos para a estatística $F$, cobrindo cinco especificações da equação (11). Um conjunto assume que a variável explicativa (ou conjunto de variáveis explicativas) é I(0) e outro assume que seja I(1).

${ }^{8} \mathrm{O}$ deflator implicito de preços foi obtido através da diferença entre a variação nominal e real (trimestre a trimestre imediatamente anterior) do PIB e PNB. Especificamente para a economia brasileira, no periodo entre 1980:1 a 1988:4, foi utilizado o IGP ${ }_{D I}$ como proxy para o deflator implícito de preços, dado a falta de informaçōes sobre PIB trimestral nominal para o período. ${ }^{9}$ PIB e PNB a preços constantes de 1996:4 dividido pela população residente (IPEA e U. S. CENSUS BUREAU, 2002).
} 
Como considerado anteriormente, o teste de existência de um relacionamento de longo prazo entre a razão dos níveis de preços $\left(P_{(A B)}\right)$ e a razão do nível de renda real per capita $\left(Y_{(A B)}\right)$ (equação (9)), e razão dos níveis de preços e a primeira diferença da razão do nível de renda real per capita $\left(\Delta Y_{(A B)}\right)$ (todas estas variáveis em logaritmo natural) dá-se em duas etapas. A primeira consiste da seleção do número ótimo da defasagem para as primeiras diferenças a serem incluídas no modelo de correção de erros (equação (11)). A segunda etapa constitui-se no cálculo das estatísticas $F$ do teste de hipótese conjunta para b1 e b2, da equação (11), e comparar o resultado com os valores críticos de Pesaran et alii (1999 p. T1) que estabelecem a fronteira inferior (I(0)) e superior (I(I)).

Para determinar a extensão da defasagem, estimou-se o modelo condicional de correção de erros (equação (11)) para as equações (9) e (10), por MQO, sem tendência linear ${ }^{10}$, considerando a extensão dos lags de 1 a 8 . A Tabela 1 mostra os resultados da extensão dos lags para as equaçōes (9) e (10). Foram utilizados os critérios de Akaike e Schwarz, denotados por AIC (Akaike Information Criteria) e SBC (Schwarz Bayesian Criteria), e a estatística do multiplicador de Lagrange (LM) para o teste de hipótese de correlação serial dos resíduos de ordem 1 e 4 (especificados na Tabela 1 como $\chi^{2}(1) \chi^{2}(4)$ ).

Tabela 1: Determinação da extensão da ordem de defasagem para as especificações das equações (9) e (10)

\begin{tabular}{c|c|c|c|c|c|c|c|c}
\hline \multirow{2}{*}{ Lag(p) } & \multicolumn{3}{|c|}{$P_{(A B) t}=\alpha_{1}+\beta_{1} Y_{(A B) t}+u_{t}$} & \multicolumn{4}{c}{$P_{(A B) t}=\alpha_{1}+\beta_{1} \Delta Y_{(A B) t}+u_{t}$} \\
\cline { 2 - 9 } & AIC & SBC & $\chi^{2}(1)$ & $\chi^{2}(4)$ & AIC & SBC & $\chi^{2}(1)$ & $\chi^{2}(4)$ \\
\hline 1 & 160,20 & 174,72 & 0,21 & $9,81^{* *}$ & 159,79 & 174,23 & 0,29 & $11,85^{* *}$ \\
2 & 162,19 & 181,45 & $7,82^{*}$ & $11,39^{* *}$ & 161,58 & 180,73 & $8,02^{*}$ & $11,69^{* *}$ \\
3 & 155,84 & 179,79 & $3,65^{* * *}$ & 7,19 & 154,82 & 178,64 & $3,53^{* * *}$ & 7,22 \\
4 & 154,42 & 183,01 & 0,33 & 4,45 & 153,18 & 181,61 & 0,37 & 5,66 \\
5 & 156,24 & 189,41 & 1,33 & 2,78 & 154,64 & 187,79 & 0,22 & 7,11 \\
6 & 157,09 & 194,79 & 0,82 & 5,70 & 154,79 & 192,14 & 1,05 & 6,95 \\
7 & 155,52 & 197,71 & 1,85 & 6,01 & 154,46 & 196,42 & 1,34 & 5,87 \\
8 & 156,03 & 202,65 & 0,29 & 3,73 & 152,64 & 198,99 & 2,31 & 7,04 \\
\hline
\end{tabular}

Fonte: Cálculos do trabalho.

${ }^{*}$ ) Rejeito a hipótese de não autocorrelação ao nível de significância de $1 \%$ ou menos;

${ }^{* *}$ Rejeito a hipótese de não autocorrelação ao nível de significância de $5 \%$ ou menos.

$\left.{ }^{\star \star \star}\right)$ Rejeito a hipótese de não autocorrelação ao nível de significância de $10 \%$ ou menos.

${ }^{10}$ A tendência linear para as duas especificações não se mostrou estatisticamente significativa. 
De acordo com a Tabela 1 , para a especificação da razão dos preços como função da razão da renda real per capita (equação (9)), a extensão do lag é 6 pelo critério de AIC e 8, pelo de SBC. Utilizando o critério da parcimônia, foi escolhido o lag igual a 6. De forma semelhante, para a especificação da equação (10), a razão dos preços como função da primeira diferença da razão da renda real per capita, o lag escolhido foi 6.

Desta forma, após a identificação do número ótimo de defasagens, estima-se a equação (11) para este nível de defasagem, e calcula-se a estatística $F$ para a hipótese conjunta da significância da variável explicativa e dependente defasadas em um período serem zero.

Desta forma, os resultados da Tabela 2 mostram que a razão do nível de preços e a razão do nível de renda real per capita (equação (9)) e a primeira diferença do nível de renda per capita (equação (10)) não apresentam um relacionamento de longo prazo, pois os valores $\mathrm{F}$ e $\mathrm{t}$ calculados são menores que os valores críticos ${ }^{11}$ para a situação I(0). Isto é, as variáveis não cointegram ${ }^{12}$. Esta inferência pode ser feita independentemente do conhecimento da ordem de integração das variáveis.

Desta forma, pode-se dizer que, para o período de tempo analisado, o efeito Balassa-Samuelson não se verifica para a economia brasileira. Assim, a premissa da PPC como uma mensuração da taxa de câmbio de equilíbrio de longo prazo pode ser considerada. Ou seja, a falha estrutural da versão relativa da PPC, em termos do crescimento econômico afetando os preços relativos de tradables e nontradables, não se verificou para o caso da economia brasileira no intervalo de tempo considerado.

Tabela 2: Cálculo das estatísticas F e t para a análise da existência de relação de longo prazo entre as variáveis

\begin{tabular}{c|c|c|c}
\hline Especificą̧ão & Lag $|p|$ & EstatísticaF & Estatísticat \\
\hline$P_{(A B) t}=\alpha_{1}+\beta_{1} Y_{(A B) t}+u_{t}$ & 6 & 1,425 & 0,853 \\
$P_{(A B) t}=\alpha_{1}+\beta_{1} \Delta Y_{(A B) t}+u_{t}$ & 6 & 1,745 & $-0,919$ \\
\hline
\end{tabular}

Fonte: Cálculos do trabalho.

\footnotetext{
${ }^{11}$ As Tabelas C1.iii e C2.iii ( PESARAN et alii. 1999, p. T2 e T4) apresentam os valores críticos da estatistica F e $t$ para I(0) e I(1) com nível de significância de $10 \%, 5 \%$ e $1 \%$. Considerando apenas um regressor, $\mathrm{k}=1$, os valores críticos com nível de significância de $1 \%$ e $5 \%$ são: $(6,84$ e 7,84) e $(4,94$ e 5,73) para $F$ e $(-3,42$ e -3,82) e $(-2,86$ e -3,22) para $t$, respectivamente.

${ }^{12}$ Estes resultados se mantêm mesmo utilizando lags 7 e 8 .
} 
De certa forma, estes resultados estão de acordo com um corpo de literatura empírica que afirma que a hipótese da PPC se mantém no longo prazo para a economia brasileira.

\section{Considerações finais}

As principais críticas à PPC se resumem nos argumentos de falta de concatenação da teoria da PPC com os fundamentos microeconômicos de flexibilização de preços e salários; verificação de imperfeições no mercado de trocas; diferenças nas estruturas de mercados, implicando diferentes ponderaçōes de índices de preços; e crescimento econômico afetando os preços relativos.

Desta forma, dada a importância da PPC nos modelos de determinação da taxa de câmbio, procurou-se verificar empiricamente a crítica a esta teoria em termos do crescimento econômico afetando os preços relativos, ou seja, testar o efeito Balassa-Samuelson. Utilizando uma abordagem de séries temporais, através do teste de fronteira desenvolvido por Pesaran, Shin e Smith (1999), verificou-se a existência de um relacionamento de longo prazo entre as variáveis razão dos níveis de preços e razão do nível de renda real per capita para a economia brasileira e a americana.

Os resultados do trabalho evidenciaram que não existe relação de longo prazo entre as variáveis, isto é, não há evidência do efeito Balassa-Samuelson no longo prazo para a economia brasileira no período de tempo considerado.

Este resultado, de certa forma, está de acordo com o esperado, visto que, na literatura sobre teste da validade da PPC para a economia brasileira, há exemplos de confirmação da hipótese da PPC. Entretanto, a rejeição do efeito BS não implica diretamente a validade da PPC, apenas que esta crítica não se confirmou. Desta forma, trabalhos futuros podem ser direcionados no sentido de testar a hipótese de que a validade da PPC implica que a taxa de câmbio real não afeta o lado real da economia.

\section{Referências Bibliográficas}

ANDRÉS, J., HERMANDDO, I. and KRUGER, M,. Growth, inflation and the exchange rate regime. Economics Letters, 53, 1996. p. 61-65. 
BALASSA, B. The purchasing power parity doutrine: a reappraisal. Journal of Political Economy. 72, 1964. p. 584-596.

BERGSTRAND, J. H. Structural determinantes of real exchange rates and national price levels: some empirical evidence. American Economic Review. 81, 1991. p.325-334.

BUREAU OF ECONOMIC ANALYSIS - BEA. National income and product account tables. U. S. Department of Commerce, 2002. Disponivel em: www.bea.doc.gov/bea/dn/gdplev.xlx. Acessado em 11/02/02).

DE GREGORIO, J.; WOLF, H. C. Terms of trade, productivity and the real exchange rate. NBER Working Paper $n^{\circ}$ 4807. 1994.

FARIA, J. R.; LEÓN-LEDESMA, M. Testeing the Balassa-Samuelson effect: implications for grawth and PPP. Working paper. Studies in Economics, Departament of Economic University of Kent- UK.Sepetenber 2000. Disponivel em: http://www.ukc.ac.uk/economics.

FAVA, V.; ALVES, D. A fractional cointegration anlysis of purchasing power parity for Brazil. In: Encontro Brasileiro de Econometria, 18, 1996, Anais... Águas de Lindóia: SBE, 1996. p. 201-210.

FUNDAÇÃO INSTITUTO BRASILEIRO DE GEOGRAFIA E ESTATIÍSTICA. Produto Interno Bruto: Brasil. Rio de Janeiro: IBGE, 1989. 74p. (Relatório Metodológico v. 9).

. Indicadores IBGE: Contas Nacionais. 2001. Disponível em: www.ibge.gov.br/ ibge/estatistica/economia/ contasnacionais. Acessado em 20/02/02).

INSTITUTO DE PESQUISA APLICADA - IPEA. IPEADATA Base de dados macroeconômicos: Séries históricas de população residente, 2002 Disponivel em: www.ipeadata.gov.br/ipeaweb.dll/Nserie. Acessado em 12/02/02.

KRAVIS, I. B. and LIPSEY, R. E. National price levels and the prices of trabables and non-tradables. American Economic Review.78, May, 1988. p. 474-478.

MACDONALD, R.; RICCI, L. PPP and the Balassa Samuelson effect: the role of the distribution sector. IMF Working Paper WP/01/38. 2001.

MENEZES, F. M.; RESENDE, M. Teste de cointegração da paridade do poder de compra para a economia brasileira: 1870-1906. Estudos Econômicos, v. 26, n. 1 , 1996. p. 51-62.

PEREIRA, P. L. V.; DUARTE, A. R. Paridade do poder de compra e paridade da taxa de juros para o Brasil: uma abordagem via cointegração multivariada. In. Encontro Brasileiro de Econometria, 13. 1991. Anais... Curitiba: SBE, 1991. p. 429-440.

PESARAN, M. I.; SHIN, Y.; SMITH, R. J. Bounds testing approaches to the analysis of long run relationships. Working paper $n^{\circ}$ 9907, February. Cambridge: Trinity College. 1999. Disponivel em: www.cam.ac.uk/dae/index.htm. Acessado 10-022002. 
ROSSI, J. W. O modelo monetário de determinação da taxa de câmbio: teste para o Brasil. Pesquisa e Planejamento Econômico, v. 26, n.2. 1996. p. 155-182.

SAMUELSON, P. A. Theoretical notes on trade problems. Review of Economics and Statistics, 46, 1964. p. 145-154.

STRAUSS, J. Relative price determination in the medium run: the influence of wages, productivity, and international prices. Southern Economic Journal. 65, 1998. p. 223-244.

U.S. CENSUS BUREAU. Historical national population estimatives. 2002. Disponível em: http://eire.census.gov/popest/archives/pre1980/popclockest.txt. Acessado em 11/02/02.

VASCONCELOS, C. R. F.; VASCONCELOS, S. P.; LIMA, R. C. Paridade do poder de compra: um teste de cointegração para o caso brasileiro. Revista Econômica do Nordeste. V. 30, N. Especial, dezembro. 1999. p. 926-936.

ZINI JÚNIOR, A. A.; CATI, R. C. Cointegração e taxa de câmbio: teste sobre a PPP e os termos de troca do Brasil dee 1855 a 1990. Pesquisa e Planejamento Econômico, v. 23, n. 2, 1993. p. 348-374. 